\title{
Investigation of Strain in Core@shell Electrocatalysts with ADF-STEM and 4D STEM Scanning Nanodiffraction
}

Debangshu Mukherjee ${ }^{*}$, Jocelyn T.L. Gamler ${ }^{2}$, Xiahan Sang ${ }^{1}$, Suhas Somnath ${ }^{3}$, Sara E. Skrabalak ${ }^{2}$ and Raymond R. Unocic ${ }^{1 *}$

1. Center for Nanophase Materials Sciences, Oak Ridge National Laboratory, Oak Ridge, USA.

2. Department of Chemistry, Indiana University Bloomington, Bloomington, USA.

3. Center for Computational Sciences, Oak Ridge National Laboratory, Oak Ridge, USA.

*Corresponding authors: mukherjeed@ornl.gov, unocicrr@ornl.gov

Strain engineering of bimetallic core@ @hell electrocatalysts is a promising approach to increase catalytic selectivity, activity, and durability of catalysts in electrochemical energy conversion applications [1,2]. The catalytic activity has been proposed to be controlled by the metal-ligand bond strengths [2,3]. Furthermore, surface strain has been demonstrated to be a controlling factor for the bond strength through modification of the metal-metal bond length and thus, strain is regarded as one of the most significant tuning variables available for enhancing catalytic activity. To better correlate strain at atomic-level interfaces with catalyst functionality, it is important to precisely measure the local lattice strain. Several approaches have been explored to quantify local strain: coherent X-ray diffraction, annular dark field (ADF) scanning transmission electron microscopy (STEM), and X-ray absorption spectroscopy among others [1-4]. Of all the available approaches, electron microscopy is by far the only technique that enables direct visualization of a catalyst nanoparticle's atomic structure, allowing for the straightforward measurement of strain directly from the positions of the atomic columns. However, in reality the expected displacements due to strain are similar to the precision limits of ADF-STEM ( 2pm). Additionally, scan and drift distortions are limiting factors in strain measurement derived from ADF-STEM datasets. Scanning nanodiffraction has been proposed as an alternative, robust technique for strain measurements, where the strain is calculated in the diffraction plane from the positions of the higher order diffraction disks [5]. Yet, depending on the disk fitting algorithms used, significant variations can be present in strain measurements, even when using the same underlying dataset.

Here, we demonstrate atomic-resolution ADF-STEM imaging and scanning nanodiffraction of bimetallic Pd@Pt core@shell nanoparticles synthesized using the seed-growth method [6]. Atomic-resolution ADFSTEM imaging and scanning nanodiffraction datasets of the same nanoparticles were collected back-toback on an aberration-corrected Nion UltraSTEM100 operated at $100 \mathrm{kV}$, with the only difference being a change in the STEM beam convergence semi-angle used between the two acquisitions. High-angle annular dark field (HAADF)-STEM datasets were collected with a convergence semi-angle of $32 \mathrm{mrad}$ (Fig. 1a) while the scanning nanodiffraction patterns were collected with a convergence semi-angle of 3.5 mrad to enable non-overlapping diffraction disks (Fig. 1b). Individual diffraction patterns were recorded using a Hamamatsu ORCA CMOS detector at 200Hz. Two different approaches are being pursued for quantitative strain measurement comparison. For HAADF-STEM datasets - fitting of atomic columns as gaussians and subsequent analysis of strain from the column positions and geometric phase analysis. For the scanning nanodiffraction datasets - fitting of diffraction disks performed both through Sobel- and Canny-type edge detection techniques and cross-correlation. This enables us to quantify strain from multiple techniques on the same particle and map the strain evolution across the shell thickness [7].

References: 
[1] D Wang et al., Nature Materials 12 (2013), p. 81.

[2] P Strasser et al., Nature Chemistry 2 (2010), p. 454.

[3] S Zhang et al., Journal of the American Chemical Society 136 (2014), p. 7734.

[4] S Manna et al., AIP Advances 7 (2017), p. 125025.

[5] TC Pekin et al., Ultramicroscopy 176 (2017), p.170.

[6] N Ortiz et al., ACS Nano 8 (2014), p. 12461.

[7] Research supported by ORNL's Laboratory Directed Research and Development Program, which is managed by UT-Battelle, LLC for the U.S. Department of Energy (DOE). Electron microscopy was conducted as part of a user proposal at Oak Ridge National Laboratory's Center for Nanophase Materials Sciences (CNMS), which is a U.S. DOE Office of Science User Facility. This research used resources of the Compute and Data Environment for Science (CADES) at the Oak Ridge National Laboratory, which is supported by the Office of Science of the U.S. DOE under Contract No. DE-AC05-00OR22725.

a)

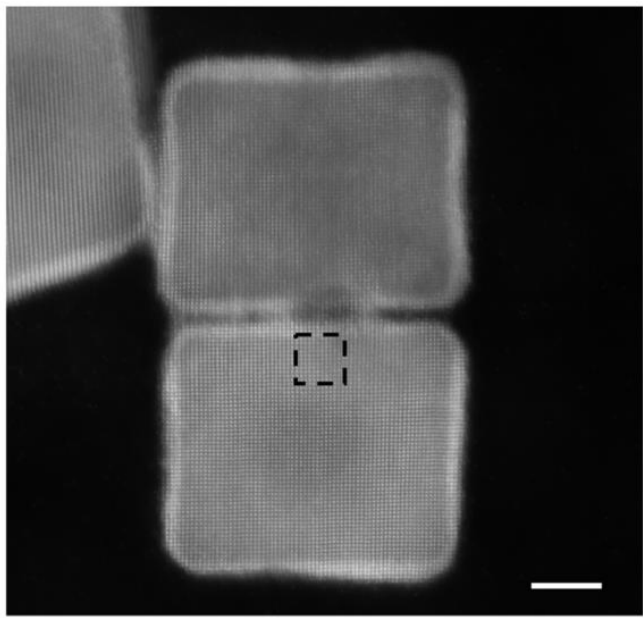

c)

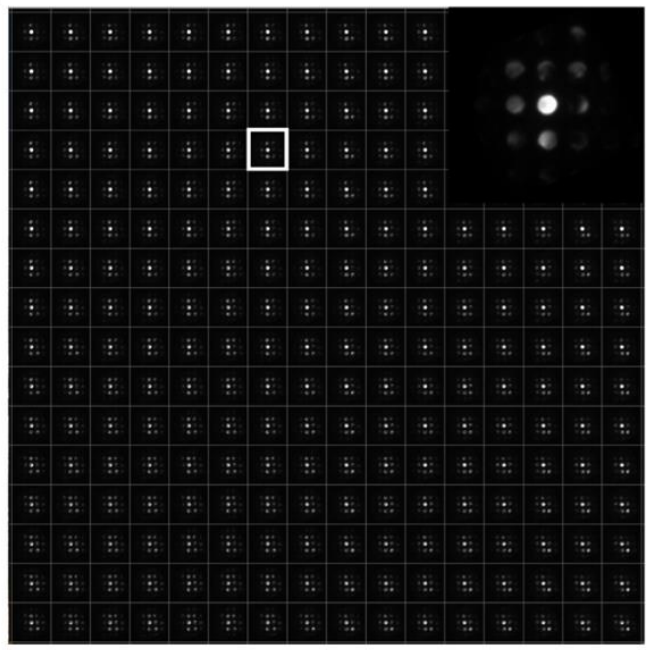

b)

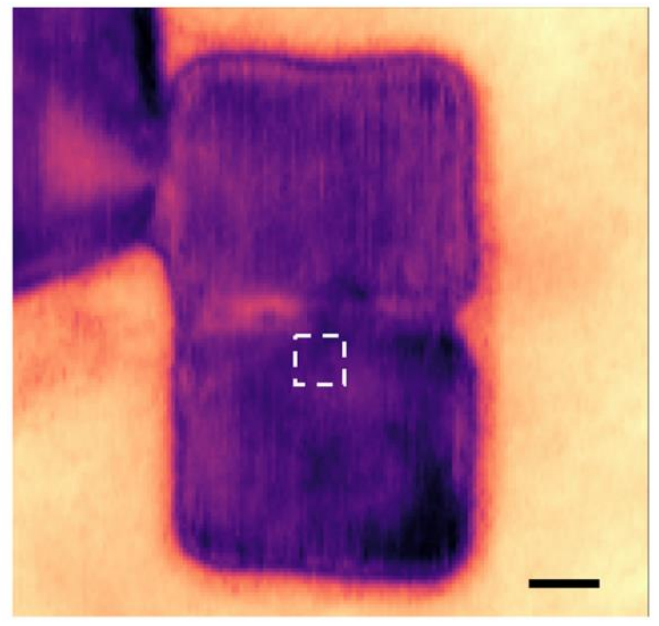

d)

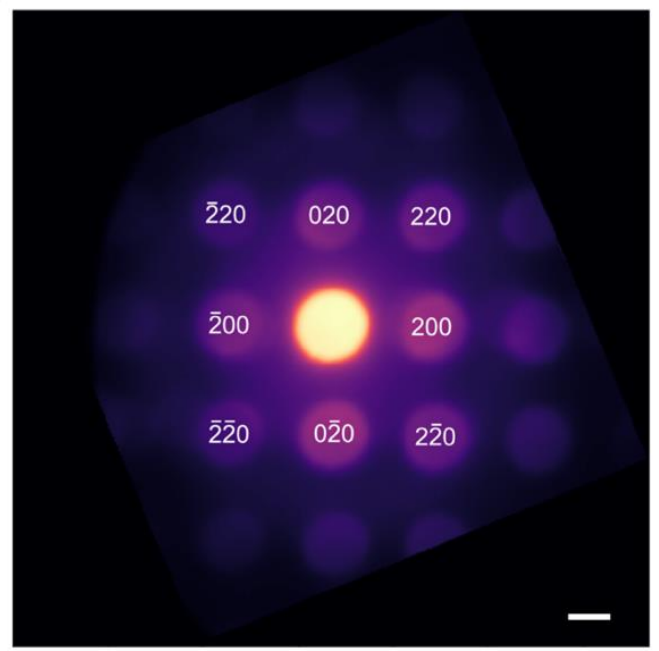

Figure 1. a) Aberration-corrected ADF-STEM imaging of Pd@Pt nanoparticles with beam convergence semi-angle of $32 \mathrm{mrad}$. Scale bar is $2 \mathrm{~nm}$. b) Sum of the zeroth order central disks of same region in (a) imaged with a $3.5 \mathrm{mrad}$ convergence semi-angle. Scale bar is $2 \mathrm{~nm}$. c) Set of nanodiffraction patterns showing bright central disk and first order diffraction disks from region of image marked by black dashed box in (a) and white dashed box in (b). Inset shows a single diffraction pattern. d) Logarithm of the mean of all diffraction patterns, with first order spots indexed. Scale bar is $3.5 \mathrm{mrad}$. 